\title{
Review
}

\section{The Status of Specialized Ambulatory Heart Failure Care in Canada: A Joint Canadian Heart Failure Society and Canadian Cardiovascular Society Heart Failure Guidelines Survey}

Writing Group: Sean A. Virani, MD, $\stackrel{\text { a, }}{ }$ Shelley Zieroth, MD, $\stackrel{\text {, }}{ }$ Sharon Bray, ${ }^{c}$ Anique Ducharme, MD, ${ }^{\mathrm{d}}$ Karen Harkness, RN, ${ }^{\mathrm{e}}$ Sheri L. Koshman, PharmD, Michael McDonald, MD, ${ }^{\mathrm{g}}$ Eileen O’Meara, MD, ${ }^{\mathrm{d}}$ Elizabeth Swiggum, MD; ${ }^{\mathrm{h}}$ on behalf of the Canadian Heart Failure Society and the Canadian Cardiovascular Society Heart Failure Guidelines

Panel; CCS HF Guidelines Primary and Secondary Panel: Michael Chan, MBBS,

Justin A. Ezekowitz, MBBCh, ${ }^{\mathrm{f}}$ Nadia Giannetti, MD, ${ }^{\mathrm{j}}$ Adam Grzeslo, MD,

George A. Heckman, MD, Jonathan G. Howlett, MD, ${ }^{\mathrm{m}}$ Serge Lepage, MD, ${ }^{\mathrm{n}}$

Lisa Mielniczuk, MD, ${ }^{\circ}$ Gordon W. Moe, MD, ${ }^{\mathrm{p}}$ Mustafa Toma, MD, ${ }^{\mathrm{a}}$ Howard Abrams, MD, ${ }^{\mathrm{q}}$

Abdul Al-Hesaye, MD, ${ }^{\mathrm{P}}$ Alain Cohen-Solal, MD, ${ }^{\mathrm{r}}$ Michel D'Astous, MD, ${ }^{\mathrm{s}}$ Sabe De, MD,

Diego Delgado, MD, ${ }^{\mathrm{q}}$ Olivier Desplantie, MD, ${ }^{\mathrm{h}}$ Estrellita Estrella-Holder, RN, ${ }^{\mathrm{u}}$ Lee Green, MD, ${ }^{\mathrm{f}}$

Haissam Haddad, MD, Adrian F. Hernandez, MD, ${ }^{\mathrm{w}}$ Simon Kouz, MD,

Marie-Hélène LeBlanc, MD, ${ }^{\mathrm{y}}$ Douglas Lee, MD, ${ }^{\mathrm{q}}$ Frederick A. Masoudi, MD, ${ }^{\mathrm{z}}$

Sylvain Matteau, MD, ,aa Robert McKelvie, MD, ${ }^{\text {bb }}$ Marie-Claude Parent, MD, ${ }^{\mathrm{d}}$

Miroslaw Rajda, MD, ${ }^{c c}$ Heather J. Ross, MD, ${ }^{\text {dd }}$ and Bruce Sussex, MBBS ${ }^{\text {ee }}$

\footnotetext{
${ }^{a}$ University of British Columbia, Vancouver, British Columbia, Canada $;{ }^{b}$ University of Manitoba, Winnipeg, Manitoba, Canada; ${ }^{c}$ Lived Experience Partner, Toronto, Ontario, Canada; ${ }^{d}$ Institut de Cardiologie de Montréal, Université de Montréal, Montréal, Québec, Canada; ${ }^{e}$ Hamilton Health Sciences, Hamilton, Ontario, Canada; ${ }^{f}$ University of Alberta, Edmonton, Alberta, Canada; ${ }^{g}$ Peter Munk Cardiac Centre, University Health Network, Toronto, Ontario, Canada; ${ }^{h}$ Royal Jubilee Hospital, University of British Columbia, Victoria, British Columbia, Canada; ${ }^{i}$ University of Alberta, Royal Alexandra Hospital, Edmonton, Alberta, Canada; ${ }^{j}$ McGill University, Montréal, Québec, Canada; ${ }^{k}$ Hamilton Health Sciences, McMaster University, Hamilton, Ontario, Canada; ${ }^{\prime}$ Schlegel-University of Waterloo Research Institute for Aging, University of Waterloo, Waterloo, Ontario, Canada; ${ }^{m}$ Cumming School of Medicine University of Calgary, Libin Cardiovascular Institute, Calgary, Alberta, Canada; ${ }^{n}$ Université de Sherbrooke, Sherbrooke, Québec, Canada; ${ }^{\circ}$ University of Ottawa Heart Institute, Ottawa, Ontario, Canada; ${ }^{p}$ St Michael's Hospital, Toronto, Ontario, Canada; ${ }^{q}$ University Health Network, Toronto, Ontario, Canada; ${ }^{r}$ Paris University, UMR-S 942, Hôpital Lariboisière, Paris, France; ${ }^{5}$ CHU Dumont UHC, Moncton, New Brunswick, Canada; ${ }^{t}$ London Health Sciences, Western University, London, Ontario, Canada, ${ }^{\prime}$ Saint Boniface General Hospital, Winnipeg, Manitoba, Canada; ${ }^{v}$ University of Saskatchewan, Saskatoon, Saskatchewan, Canada; ${ }^{w}$ Duke Clinical Research Institute, Durham, North Carolina, USA; ${ }^{x}$ Centre Intégré de Santé et de Services Sociaux de Lanaudière - Centre Hospitalier de Lanaudière, Joliette, Québec, Canada; ${ }^{y}$ Institut universitaire de cardiologie et de pneumologie de Québec (IUCPQ), Université Laval, Québec, Québec, Canada; ${ }^{z}$ University of Colorado Anschutz Medical Campus, Aurora, Colorado, USA; ${ }^{a a}$ Chaleur Regional Hospital, Bathurst, New Brunswick, Canada; ${ }^{b b}$ St Joseph's Health Care, Western University, London, Ontario, Canada; ${ }^{c c}$ QEII Health Sciences Centre, Halifax, Nova Scotia, Canada; ${ }^{d d}$ Peter Munk Cardiac Centre, University Health Network, Toronto, Ontario, Canada; ${ }^{e e}$ Memorial University, St John's, Newfoundland, Canada
}

Received for publication January 30, 2020. Accepted March 4, 2020.

Ethics Statement: We used the ARECCI Tool which confirmed this project to be a quality improvement initiative, and thus no formal ethics approval was required.
${ }^{\ddagger}$ Co-lead authors.

Corresponding author: Dr Shelley Zieroth, Section of Cardiology, University of Manitoba, Winnipeg, Manitoba, Canada.

E-mail: szieroth@sbgh.mb.ca

See page 159 for disclosure information. 


\begin{abstract}
This joint Canadian Heart Failure Society and the CCS Heart Failure guidelines report has been developed to provide a pan-Canadian snapshot of the current state of clinic-based ambulatory heart failure (HF) care in Canada with specific reference to elements and processes of care associated with quality and high performing health systems. It includes the viewpoints of persons with lived experience, patient care providers, and administrators. It is imperative to build on the themes identified in this survey, through engaging all health care professionals, to develop integrated and shared care models that will allow better patient outcomes. Several patient and organizational barriers to care were identified in this survey, which must inform the development of regional care models and pragmatic solutions to improve transitions for this patient population. Unfortunately, we were unsuccessful in incorporating the perspectives of primary care providers and internal medicine specialists who provide the majority of $\mathrm{HF}$ care in Canada, which in turn limits our ability to comment on strategies for capacity building outside the HF clinic setting. These considerations must be taken into account when interpreting our findings. Engaging all HF care providers, to build on the themes identified in this survey, will be an important next step in developing integrated and shared care models known to improve patient outcomes.
\end{abstract}

The purpose of this report is to provide a pan-Canadian snapshot of the current state of clinic-based ambulatory heart failure (HF) care in Canada with specific reference to elements and processes of care associated with quality and high performing health systems. It includes the viewpoints of persons with lived experience, patient care providers, and administrators. Canada is uniquely poised to undertake this survey due to a strong culture of collaboration within the HF community. Herein, we present the results of a national survey informed by Canadian HF key opinion leaders including Primary and Secondary Canadian Cardiovascular Society (CCS) HF Guidelines Committee members and broader representation from Canadian HF care providers including members of the Canadian Heart Failure Society (CHFS).

This work product is intended to serve as a national source document for current practice related to HF multidisciplinary care and is developed primarily with cardiologists and specialty HF care providers in mind. It builds on the recommendations and commentary presented in the 2017 Comprehensive Update of the CCS HF Guidelines and the 2016 HF Companion $^{1,2}$ by reporting on the national landscape of HF care models. Through a better understanding of how health services are being delivered for this high-risk group of patients, we can better articulate comprehensive local, provincial, and national strategies to improve care processes and outcomes. As such, this document aims to serve as a starting point to define the desired future state for HF service delivery in Canada.

\section{RÉSUMÉ}

Ce rapport conjoint des lignes directrices de la Société canadienne d'insuffisance cardiaque et de la Société canadienne de cardiologie (SCC) sur l'insuffisance cardiaque a été élaboré pour fournir un aperçu pancanadien de l'état actuel des soins ambulatoires de l'insuffisance cardiaque (IC) en clinique au Canada, en se référant spécifiquement aux éléments et aux processus de soins associés à des systèmes de santé très performants et de qualité. Il comprend les points de vue de personnes ayant une expérience vécue de l'IC, de prestataires de soins aux patients et d'administrateurs. Il est impératif de s'appuyer sur les thématiques identifiées dans cette enquête, en y engageant tous les professionnels de la santé, pour développer des modèles de soins intégrés et partagés qui permettront de meilleurs pronostics pour les patients. Plusieurs obstacles relatifs aux patients et organisationnels dont il faudra se soucier ont été identifiés dans cette enquête, qui doit servir de base à l'élaboration de modèles de soins régionaux et de solutions pragmatiques pour améliorer les transitions pour cette population de patients. Malheureusement, nous n'avons pas réussi à intégrer les points de vue des prestataires de soins primaires et des spécialistes en médecine interne qui fournissent la majorité des soins en IC au Canada, ce qui limite notre capacité à commenter les stratégies de renforcement des capacités en dehors du cadre des cliniques d'IC. Ces considérations doivent être prises en compte lors de l'interprétation de nos conclusions. L'engagement de tous les prestataires de soins de santé en IC à s'appuyer sur les thématiques identifiées dans cette enquête constituera une prochaine étape importante dans le développement de modèles de soins intégrés et partagés connus pour améliorer le pronostic des patients.

\section{Methods}

Key references and data sources include international best practice papers, high-performing provincial models of HF care (eg, CorHealth Ontario's Spoke-Hub-Node Model, Health Quality Ontario's Quality Based Procedures, and Cardiac Services BC's Tiers of Service Framework), ${ }^{1,3-7}$ and opinion obtained by polling all members of the CCS HF guidelines panel, CHFS members, and national HF leaders. The primary and secondary panel members collaboratively identified the need for the survey, participated where appropriate, and reviewed the manuscript. The writing group was responsible for analysis of the survey data and composition of manuscript. An online survey request (Supplemental Appendix S1) was sent to $45 \mathrm{HF}$ clinics in Canada from September 2018 to December 2018. Unique identifiers of each program were assigned to avoid duplicate data collection. The survey respondents predominantly consisted of self-identified tertiary and quaternary perspectives. All $45 \mathrm{HF}$ clinics responded; however, only 36 completed the full survey (from all Canadian provinces except PEI, and also excluding Yukon, Nunavut, and the North West Territories). Despite our attempts to engage the full spectrum of HF care providers, the primary care provider (PCP) viewpoint is not sufficiently represented in this report (Table 1). Informants were encouraged to engage with their local teams and other stakeholders, before responding to the survey, to ensure that the best data, knowledge, and perspectives were included. The survey focused on 4 broad areas of clinic-based ambulatory 


\begin{tabular}{|c|c|c|c|c|c|c|c|c|c|c|}
\hline \multicolumn{10}{|c|}{ Descriptor } & \multirow[b]{3}{*}{$\%(\mathrm{~N})$} \\
\hline \multirow[b]{2}{*}{ Type of clinic } & \multicolumn{9}{|c|}{ Location by province } & \\
\hline & $\mathrm{BC}$ & $\mathrm{AL}$ & SK & $\mathrm{MB}$ & $\mathrm{ON}$ & QC & NL & NB & NS & \\
\hline Tertiary care outpatient clinic & 4 & 3 & 1 & 1 & 8 & 3 & 1 & 1 & 1 & $51.1(23)$ \\
\hline Quaternary care outpatient clinic & 1 & 1 & - & - & 3 & 7 & - & - & 1 & $28.8(13)$ \\
\hline $\begin{array}{l}\text { Chronic disease } \\
\text { management/complex care clinic }\end{array}$ & - & - & 1 & - & 2 & 1 & - & - & - & $8.8(4)$ \\
\hline Primary care setting & - & 1 & - & - & - & - & - & 1 & - & $4.4(2)$ \\
\hline Community health clinic & - & - & - & - & - & 1 & - & - & - & $2.2(1)$ \\
\hline $\begin{array}{l}\text { Other (hospital, } \\
\text { cardiology clinic, solo practice) }\end{array}$ & - & - & - & 1 & - & 1 & - & - & - & $4.4(2)$ \\
\hline
\end{tabular}

HF care, specifically (1) human and structural resources, (2) processes of care, (3) transitions in care, and (4) quality of care.

\section{Current State: Models of HF Clinic (HFC)-Based Care in Canada}

The majority of respondents self-identified as tertiary $(\mathrm{n}=$ 24) or quaternary $(\mathrm{n}=12) \mathrm{HF}$ care) centres. Therefore, much of what we were able to ascertain about the current state of Canadian HF services reflects activity in higher intensity and more fully resourced practice settings.

HFC referrals are typically received from a variety of sources including hospital in-patient settings (most common), urgent care centres, and specialist offices. More than half of all programs surveyed $(58 \%)$ will accept patient referrals from PCPs. A majority of referrals to tertiary and quaternary HF clinics are received at the time of or after hospital discharge, whereas a lesser number are triggered by an emergency department visit (Fig. 1). Notably, natriuretic peptide levels are felt to be an important or very important tool for triage and follow-up among $65 \%$ of respondents; however, $25 \%$ of HFCs do not have access to this important biomarker.

There is also heterogeneity with respect to HFC admission criteria. Although a slight majority $(56 \%)$ of HFCs have welldefined criteria for patient enrolment, a significant proportion of clinics (40\%) will accept referrals irrespective of baseline clinical status. The most common reasons for declining or redirecting an HFC referral is failure to meet prespecified clinical criteria at the time of referral. For patients who are not felt to be appropriate for HFC assessment, individual programs will either refer to an alternative clinic, redirect to the primary cardiologist/internist, or redirect the PCP to identify more appropriate resources to support the patient's care needs.

Furthermore, patients newly discharged from hospital, after an HF exacerbation, had variable access to the surveyed HFCs with only $51 \%$ of respondents accepting any discharged patient. It is well established that this patient population is particularly vulnerable, with 30-day readmission rates reported in the literature as high as $25 \% .^{8-13}$ Both preprinted order sets and patient-centred discharge tools were identified as important components of transitional care processes. ${ }^{14}$ The transition elements most frequently used as part of these local and regional pathways included follow-up appointments booked at the time of discharge $(73 \%)$, medication reconciliation $(68 \%)$, educational materials (51\%), patient-oriented discharge tools
(46\%), appointment reminders (43\%), interprofessional communication tools $(39 \%)$, and a patient passport (32\%). Many of these tools are well-identified components of HF transitional care services and broadly adopted as best practices (Table 2 and Supplemental Table S1). ${ }^{15,16}$

A key finding of our survey is a lack of consistency in identifying the group of patients deemed to be most appropriate for HFC services. This phenomenon has been recently described in the Canadian context ${ }^{17}$ and relates to the following factors:

- A mismatch between HFC entry criteria and a given patient's clinical risk profile.

- The perceived ability of a referred patient to benefit from HFC services and adhere to a prescribed care pathway.

- The practical challenges and resource intensity of managing multimorbid frail patients.

- A selection bias towards younger patients who may benefit from interventions and advanced therapies.

- A perception that HFCs are not an appropriate venue for the initiation of or transition to a supportive care approach.

Owing to their clinical complexity, patients with HF may have multiple touch points with the health care system (Supplemental Fig. S1). This can contribute to fragmented care, difficulty navigating care providers, and poor patient/ caregiver experiences. ${ }^{18}$ Patient-centred and seamless care requires integration of services and collaboration between care providers, as defined by the shared-care model. ${ }^{18,19} \mathrm{~A}$ substantial proportion of patients, attending the HFCs represented in this survey, do not have a PCP. In fact, less than half of all clinics identified a PCP for $>75 \%$ of their patients. Even when a consistent PCP was identified, only $21 \%$ (9 of 43) of HFCs indicated that the majority of patients enrolled in the clinic had an established shared-care management strategy between the clinic and PCP. Shared care, when identified, was facilitated by collaborative discharge planning between care providers $(36 \%)$, nursing collaborations $(46 \%)$, and nurtured by interdisciplinary continuing medical education events (24\%). Only $24 \%$ of HFCs surveyed identified an existing Provincial HF care pathway.

Most HFCs will discharge patients (93\%) and report having well-defined HFC discharge criteria (88\%); however, more than $30 \%$ of clinics do not reliably adhere to their own 


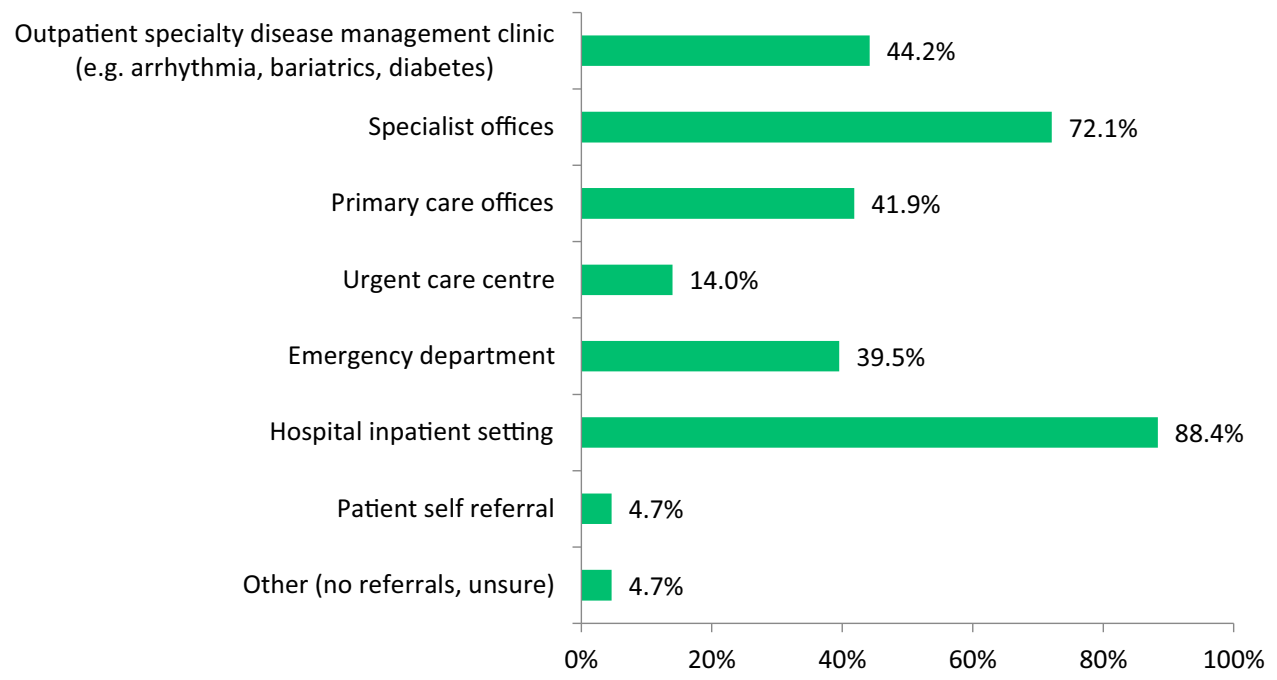

Figure 1. Accepted referral sources by clinics across Canada. The figure depicts the distribution and percentage of referral sources to heart failure clinics in Canada.

prespecified criteria, which is plausibly due to a combination of logistical challenges, system level inertia, limited community-based human resources, and infrastructure as well as patient and care provider preferences. Irrespective of the HFC setting, when patients are discharged, they are typically repatriated to their primary cardiologist/internist but can be referred back to the HFC as needed.

\section{HFC Services}

By definition, HFCs offer multidisciplinary care and a range of services; however, there is no consensus or standard as to the depth and breadth of services that should be available to all patients/caregivers enrolled in a tertiary or quaternary Canadian HFC.

The physician's role in an HFC varies depending on the practice setting. According to survey respondents, direct examination and review of every patient by a physician occurs in half $(51 \%)$ of HFCs, whereas in some clinics, the physician may perform direct in-person assessments only on an "as-needed" basis. In approximately 30\% of HFCs, patients are assigned to a single HF physician, whereas sharing of patients by a group of HF physicians within the same clinic is the more common approach (reported by $51 \%$ of HFCs).

Figure 2 highlights the percentage of Canadian HFCs that offer key services in alignment with CCS HF Guidelines and best practices. ${ }^{1}$ Almost universally, HFCs offer optimization of guideline directed medical therapy (98\%), medication, and dietary counselling $(95 \%$ and $85 \%$, respectively), and a majority of clinics also provide advance care planning $(78 \%)$, selfmanagement and education resources (73\%), and access to cardiac rehabilitation services $(68 \%)$.

Although nearly all HFCs (98\%) can offer nursing support by telephone, one-half $(51 \%)$ of HFCs routinely offer remote monitoring of patients. Remote monitoring strategies are most commonly telephone-based, although a small number of clinics also leverage implantable device diagnostics $(n=6)$ and/or web- or app-based technologies $(n=5)$.
To support the critical functions of medication titration and patient education, there is considerable practice heterogeneity. In many settings, nurse clinicians assume primary responsibility after initial consultation with an HF cardiologist; in other settings, the primary cardiologist, internist, or primary care physician assumes this responsibility through a shared-care approach. Only 14\% of survey respondents felt that a cardiologist in the HFC was the most appropriate person to complete medication titration and there is now a call for pharmacists to be more actively involved in medication adjustments. ${ }^{1,20,21}$ In our survey, key patient factors felt to be important in determining which health care provider should be responsible for ongoing pharmacologic management included frequency of hospitalization, symptom burden,

Table 2. Percentage of clinics providing services to support transitions in $\mathrm{HF}$ care

\begin{tabular}{lc}
\hline \hline Support services & $\%(\mathrm{~N})$ \\
\hline $\begin{array}{l}\text { Optimization of HF medical therapies } \\
\text { before ICD/CRT referral }\end{array}$ & $97.6(40)$ \\
Medication support and counselling & $95.1(39)$ \\
Medication reconciliation & $87.8(36)$ \\
Dietary nutrition counselling & $85.4(35)$ \\
Involve patients in shared clinical & $80.5(33)$ \\
$\quad$ decision making & \\
Advanced care and end of life planning & $78.0(32)$ \\
Self-management services and & $73.2(30)$ \\
$\quad$ resources & \\
Education sessions & $68.3(28)$ \\
Exercise training and support/cardiac & $68.3(28)$ \\
$\quad$ rehab & $36.6(15)$ \\
Smoking cessation program & $34.1(14)$ \\
Counselling services & $31.7(13)$ \\
Online tools and education & $29.3(12)$ \\
Influenza vaccinations & $12.2(5)$ \\
Patient support group & $04.9(2)$ \\
Caregiver support group &
\end{tabular}

CRT, cardiac resynchronization therapy; HF, heart failure; ICD, implantable cardiac defibrillator. 


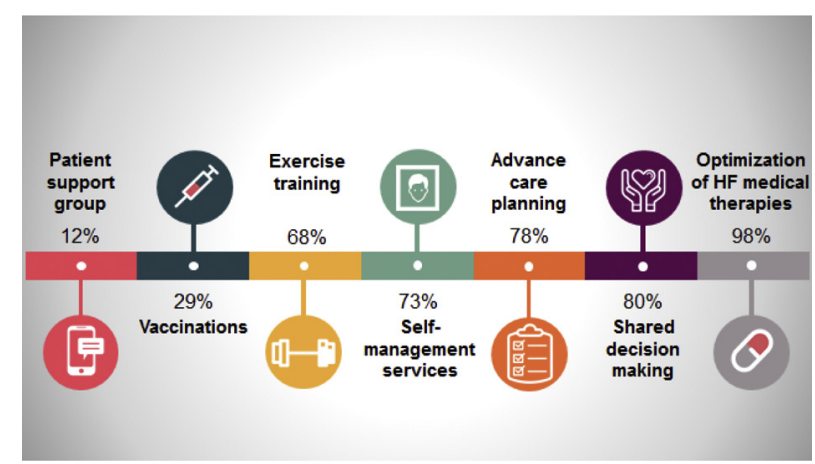

Figure 2. Percentage of clinics offering specific heart failure (HF) services.

ejection fraction, consideration for advanced therapies, and consideration for "novel" therapies.

Similarly, primary responsibility for patient education varies nationally. These services may be delivered by HFC nurses, physicians, and/or other allied health care professionals. Depending on available resources, patient education may be offered via one-on-one counselling (90\%), formal group education $(24 \%)$, telehealth counselling $(25 \%)$, or webbased education (20\%).

Among programs that perform assessments for mechanical circulatory support or transplantation, approximately twothirds carry out this work under the broader umbrella of the HFC, whereas one-third of these programs have a separate and dedicated advanced HFC. Average self-reported transplant volumes per centre in Canada ranged from 10 to 45 transplants/year and durable left ventricular assist device implant volumes ranged from 5 to 30 implants/year. Currently, only $45 \%$ of left ventricular assist device implanting centres provide "destination therapy" for transplant ineligible patients. The findings from our survey suggest that a range of advanced HF services are, and should be, offered in tertiary/quaternary HFC programs. At a minimum, all tertiary and quaternary HF programs should be resourced to provide timely access to assessment for transplantation and mechanical circulatory support among eligible patients, per CCS HF Guidelines. ${ }^{1}$

\section{Quality of HF Care}

According to survey respondents, approximately two-thirds of HF clinics were meeting CCS-recommended wait-time benchmarks. ${ }^{1}$ Of those who could not, the majority struggled with capacity for semiurgent to emergent patients. Table 3 lists the organization and patient-related barriers to timely access in the opinion of the survey respondents. Multiple patient level barriers were identified with the most common being physical distance and logistics related to travel. System and organizational level barriers to patient access included lack of nursing resources, administrative support, and clinic space. At least $16 \%$ of patients with HF have wait times $>2$ weeks for a semiurgent or urgent appointment as defined by CCS HF Guidelines, ${ }^{1}$ which is particularly relevant given that readmission rates approach $25 \%$ at 30 days post-HF hospitalization, ${ }^{22}$ underscoring the urgency for timely assessment and management.
Table 3. Barriers to clinic access

\begin{tabular}{ll}
\hline \hline Patient-related & \multicolumn{1}{c}{ Organizational-related } \\
\hline $\begin{array}{l}\text { Location (distance to } \\
\quad \text { clinic, transport) }\end{array}$ & Wait time \\
$\begin{array}{l}\text { Too sick } \\
\text { Advanced age/frailty }\end{array}$ & $\begin{array}{l}\text { Limited heart failure nurses } \\
\text { Lack of staff support } \\
\text { (administrative) }\end{array}$ \\
$\begin{array}{l}\text { Cost (parking, off-work, travel, } \\
\text { lodging) }\end{array}$ & Unavailable clinic rooms/space \\
No time/not a priority & Inadequate operational funding \\
Prefer to be seen by cardiologist & Location/distance/transport access \\
Too well & No after hours \\
Language barrier & No electronic medical record \\
\hline
\end{tabular}

A systemic lack of data infrastructure is a major organizational barrier to timely access to treatment. Approximately $70 \%$ of clinics surveyed use an electronic medical record; unfortunately, regular mail or fax remains the predominant mode of communication with other care providers. Surprisingly, despite significant advances in health informatics, approximately $70 \%$ of respondents rely on a faxed discharge summary to communicate with members of the health care team at the time of clinic or hospital discharge. Electronic records enable reporting of hard outcomes such as mortality, hospitalization, and readmission rates at many centres. However, data on process of care measures, including optimization of guideline-directed medical therapy and/or device therapy, are less available. In 37\% of clinics represented in this survey, there was no ability to track outcomes, highlighting the need for a comprehensive provincial and/or national HF informatics strategy. ${ }^{23}$

In general, there was excellent access to cardiac support services and consultative care, which may reflect the fact that most of our respondents were from tertiary and quaternary care centres (Fig. 3). By contrast, access to noncardiac subspecialties such as endocrinology and haematology were less timely. This care gap is significant to note given the increasing focus on noncardiovascular comorbidity burden and emerging HF clinical trial data related to diabetes, iron deficiency anaemia, and amyloidosis. ${ }^{24-26}$ Expanding the care team to encompass multiple specialty services through a person-centric model will be necessary to integrate the findings of pivotal clinical trials. Despite being firmly engrained in CCS Guidelines for more than a decade, only $66 \%$ of respondents reported access to secondary prevention programs including smoking cessation. Notably, only $69 \%$ of clinics reported timely access to palliative care services and $46 \%$ to geriatric services, which should most certainly be a target for improvement given the burden of HF and its prognosis, particularly among frail older patients.

It is reassuring that the majority of clinics represented in this survey $(85 \%)$ participate in quality improvement activities, which include quality indicator audits, clinical audits, patient satisfaction surveys, and case reviews. Patient engagement in quality assurance and review occurred in only $12 \%$ of these clinics. Most clinics did not have performance targets for process of care measures and patient self-efficacy behaviours (Table 4).

Patient-centred system performance appears to be a significant opportunity and target for quality improvement initiatives moving forward. There is a universal lack of ethnic and 


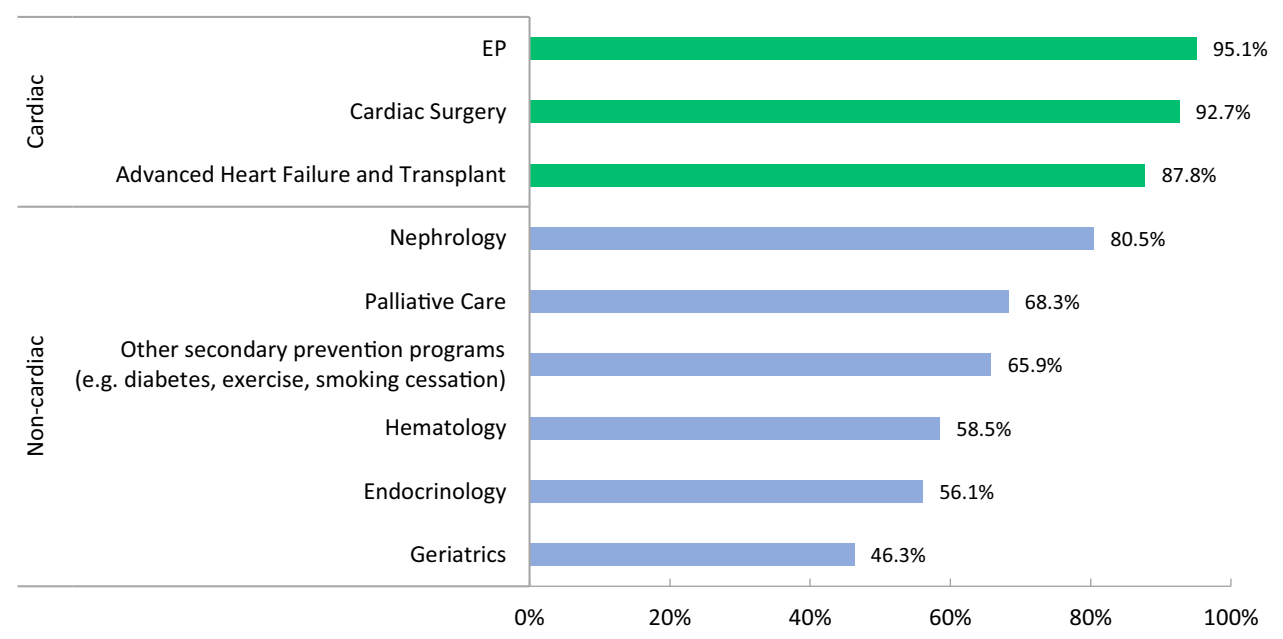

Figure 3. Patient access to specialty services. The figure demonstrates the percentage of clinics that have access to further specialty services. $\mathrm{EP}$, electrophysiology.

culturally sensitive educational materials, and the composition of HFC staff does not subjectively reflect the diversity of its constituents (Fig. 4, B-D). Clinic staff have limited knowledge to support special populations including indigenous and LGBTQ2 + communities as well as those seeking complimentary or alternative therapies (Fig. 4A). Most clinics (90\%) have some strategy in place to enable patients/caregivers to ask relevant questions and understand their care plan, which range from providing educational material in advance of a clinic visit to online support groups (Fig. 4E). There appears to be great heterogeneity in the content and quality of available educational and practice tools; given their near ubiquitous use, there would be utility in the curation of a national toolkit to ensure consistency of message and alignment with best practice. More than half of clinics did not promote or advertise patient advocacy activities (Fig. 4F). In addition, although the value and efficacy of peer-to-peer mentoring in HF populations is well established, access to patient support groups was very limited ( $12 \%$ in person vs $5 \%$ online). ${ }^{18}$

Infrastructure to support the collection, reporting, and comparison of clinical, system, and patient-reported outcome measures are consistently lacking, but are absolutely required to enable evidence-based practice, to benchmark CCS quality indicators, and to monitor and evaluate the quality and effectiveness of all HFCs in Canada.

\section{Human Resources}

There has been much discussion within the HF community globally as to the ideal complement and composition of interprofessional health care providers, which forms the multidisciplinary team at the core of chronic disease management programs. Furthermore, there are no formal recommendations as to the number and type of full-time equivalents (FTEs) essential for efficient and effective operationalization of an ambulatory HFC. Our survey provides some guidance on the current state, but not the ideal state.

An international survey of advanced HF programs (81\% from the United States) reported an annual average of 1641 patient visits per HFC per year. Our survey suggested higher
HFC volumes in Canada with an average 2496 HFC visits per clinic per year (Fig. 5). ${ }^{3}$

In the international survey, HF programs were grouped into tertiles (small, medium, and large) based on the number of staff employed. By these definitions, there are no large centres in Canada. Across all programs included in the international survey, the average number of physician FTEs per clinic was 2.65, which would suggest a ratio of 1 medical doctor per 619 patient visits. Accurate comparative Canadian data could not be obtained through our survey. The average number of nursing and other health care professional FTEs per clinic, in both the Canadian and international context, is shown in Table 5.

Among Canadian HFCs, Registered Nurses and Advance Practice Nurses are most frequently employed as a part of the

Table 4. Percentage of heart failure clinics surveyed with performance targets of any kind for care and self-efficacy behaviours

\begin{tabular}{lc}
\hline \hline Performance target & Percentage \\
\hline $\begin{array}{l}\text { Self-efficacy } \\
\text { Home weight monitoring and } \\
\quad \text { biometric data documentation }\end{array}$ & 35.0 \\
$\quad$ Patient self-management plans & 44.7 \\
Process of care & \\
$\quad$ Eligible patients prescribed RAASi & 53.6 \\
$\quad$ and $\beta$-blockers & \\
$\quad$ Advanced care plans & 29.0 \\
influenza vaccination rates & 34.2 \\
$\quad$ Patients referred to cardiac rehab & 27.8 \\
$\quad$ Smoking cessation rates & 25.0 \\
Clinical outcomes & \\
$\quad$ Patients visiting the emergency & 44.7 \\
$\quad$ department since last & appointment \\
Patients admitted to hospital since & \\
$\quad$ last appointment & 61.0 \\
Patient satisfaction & \\
Patient QOL assessment, eg, & 25.0 \\
$\quad$ MLWHF score & 10.8 \\
\hline
\end{tabular}

MLWHF, Minnesota Living with Heart Failure Questionnaire; QOL, quality of life; RAASi, Renin-Angiotensin System inhibitors. 
A

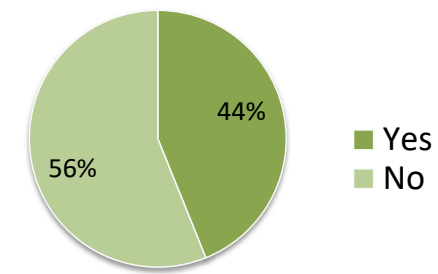

Self reported knowledge competency among HF staff to support special population

C

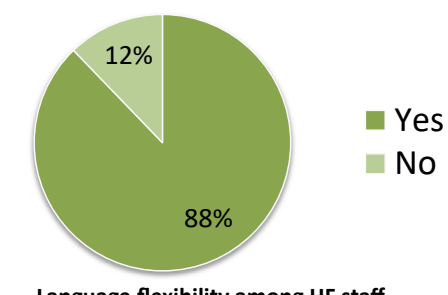

E

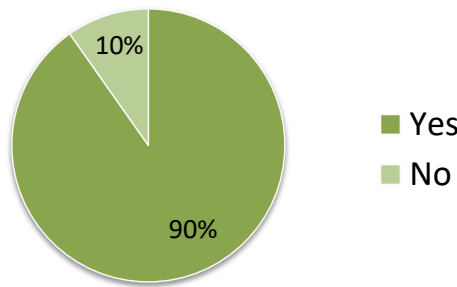

Strategies in place to help patients ask relevant questions and understand their care plan
B

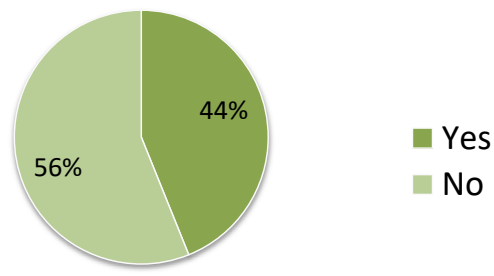

Culturally varied staff to reflect patients' cultural diversity

D

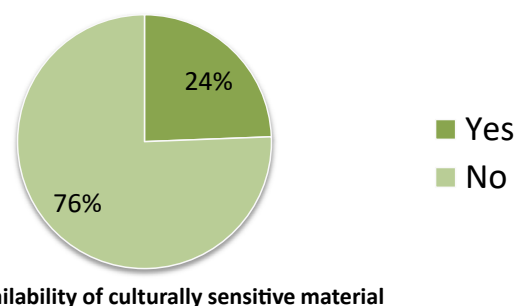

$\mathbf{F}$

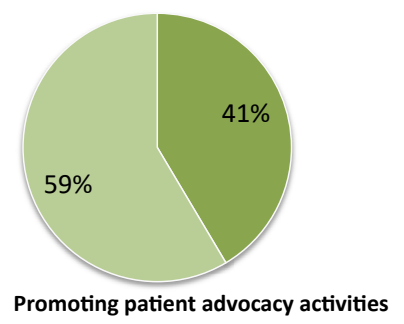

Figure 4. Prevalence of patient-centred approaches among heart failure (HF) clinics.

nonphysician team. The average staffing complement was 2.31 Registered Nurse FTEs, and/or 1.67 Registered Practical Nurse FTEs, and/or 2.83 Advanced Practice Nurse FTEs, and/or 1.25 Nurse Practitioner FTEs, 0.70 allied health care practitioner FTEs, and 1.60 administrative support (eg, secretarial, booking clerk, etc.) FTEs. Quaternary HFCs employ Registered Nurses more frequently, whereas tertiary HFCs have a greater reliance on Advance Practice Nurses.

There was little heterogeneity among small-sized $(\leq 4$ nonphysician staff) and medium-sized programs ( $>4$ nonphysician staff) in the number of nonphysician FTEs employed; however, medium-sized programs had overall higher staffing complements. Of note, medium-sized programs accommodated nearly 5 -fold more visits than small-

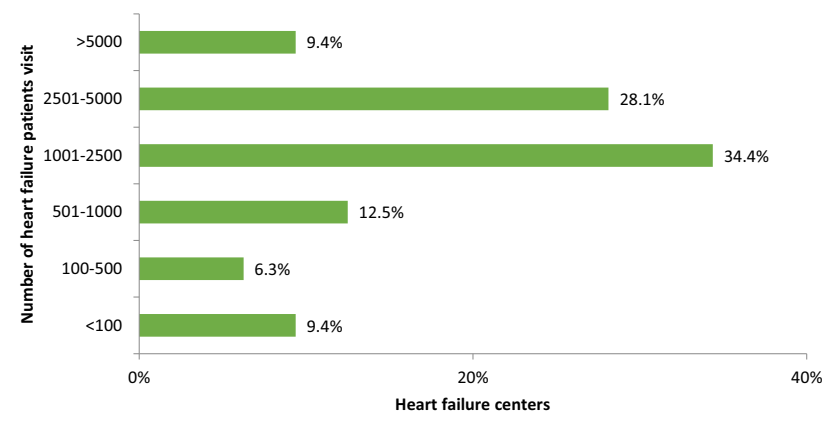

Figure 5. Percentage of respondent heart failure (HF) clinics according to the number of $\mathrm{HF}$ visits per year. sized programs, but the overall human resource complement did not follow this trend. This perhaps explains why $64 \%$ of medium-sized programs were able to meet Canadian standards for wait times as compared with $73 \%$ wait-time compliance among small-sized programs.

Tables 5 and 6 stratify Canadian HFCs by size and indicate what percent of these clinics are resourced by the various provider types. In general, a similar percentage of small- and medium-sized clinics employed nurses and nurse practitioners. However, pharmacists, registered dieticians, and other allied health care providers were less frequently employed by smallsized program compared with medium-sized programs.

The survey data demonstrate that the vast majority (95.6\%) of tertiary and quaternary Canadian HFCs are staffed by cardiologists; a few clinics have linkages with other subspecialties such as internal medicine or geriatric medicine. In all cases, physicians were actively engaged in the management of patients. In $51 \%$ of cases, physicians also provided telephone support to nurses and community-based allied health providers.

Most physicians achieved HF competency through a formal or ad hoc fellowship $(59.54 \%$ and $21.95 \%$, respectively), although a paucity of providers have formal HF certification ( 5 of 41 respondents). Only 1 respondent was Area of Focused Competence certified by the Royal College of Canada, which clearly presents an opportunity for expanded efforts at credentialing HF experts practicing in Canada. In contrast, $34 \%$ of multidisciplinary team members have professional association certification in HF. For these individuals, competency is maintained through a variety of mechanisms 
Table 5. Average Canadian nonphysician staffing by practice setting

\begin{tabular}{|c|c|c|c|c|c|}
\hline Health care provider & $\begin{array}{l}\text { Average FTEs across } \\
\text { centres }(\mathrm{n}=36)\end{array}$ & $\begin{array}{l}\text { Quaternary care } \\
\text { clinic }(\mathrm{n}=12)\end{array}$ & $\begin{array}{l}\text { Tertiary care outpatient } \\
\text { clinic }(\mathrm{n}=21)\end{array}$ & $\begin{array}{l}\text { Chronic management } \\
\text { clinic }(\mathrm{n}=3)\end{array}$ & $\begin{array}{l}\text { Reference: US } \\
\text { average FTEs }^{3}\end{array}$ \\
\hline $\begin{array}{l}\text { Average number } \\
\text { of patients' visits in the } \\
\text { previous year }\end{array}$ & - & 4601 & 2126 & 1233 & - \\
\hline Registered nurse* & 2.31 & 3.07 & 2.09 & 1.65 & 2.61 \\
\hline Registered practical nurse* & 1.67 & 2.00 & 1.00 & NR & - \\
\hline Advanced practice nurse* & 2.83 & NR & 3.00 & 2.50 & - \\
\hline Nurse practitioner* & 1.25 & 1.50 & 1.20 & 0.50 & 2.21 \\
\hline Allied health care practitioner & 0.70 & 0.84 & 0.62 & 0.43 & $0.53^{\dagger}$ \\
\hline Pharmacist & 0.76 & 1.15 & - & - & 0.59 \\
\hline Registered dietitian & 0.72 & 0.79 & 0.72 & 0.30 & 0.75 \\
\hline $\begin{array}{l}\text { Any administrative support (eg, } \\
\text { secretarial, booking, etc) }\end{array}$ & 1.60 & 1.66 & 1.81 & 1.00 & $0.47^{\ddagger}$ \\
\hline
\end{tabular}

FTE, full time equivalent; NR, not reported.

* Nursing roles are defined by scope of practice and level of education attained. For more information, including definition, please visit https://www.cna-aiic.ca.

${ }^{\dagger}$ Average FTEs of allied health care staff including registered dietitian, social worker, physical therapist, and psychologist.

$\ddagger$ Reported for financial consultants.

including webinars, local meetings, and annual conferences. It should be noted that continuing professional development funding for nonphysician HF care providers is limited and appears to be diminishing; thus knowledge translation activities must be tailored with this in mind and strategies for enhanced funding to support ongoing learning pursued. This is particularly relevant because our survey would suggest that HFCs rely heavily on nonphysician FTEs to provide the bulk of HF care in Canada.

\section{Conclusion}

The survey results presented here are the first attempt to comprehensively describe the current status of ambulatory clinic-based HF care in Canada. These data, the questions raised, and the care gaps identified are intended to inform a national dialogue on what optimal HF care should look like and frame our understanding of how the desired future state can be realized. It is imperative that Canada remains in step with global trends and initiatives in HF care and that our HF leadership continues to advocate for new policies to support equitable access to HF services. These types of initiatives must be supported by measurement and evaluation, which will drive our approach to quality assurance and quality

Table 6. Average Canadian nonphysician staffing by practice size (small program $\leq \mathbf{4}$ staff, medium program $5-10$ staff)

\begin{tabular}{lcc}
\hline \hline & $\begin{array}{c}\text { Small program } \\
(\mathrm{n}=11)\end{array}$ & $\begin{array}{c}\text { Medium program } \\
(\mathrm{n}=22)\end{array}$ \\
\hline Total patient visits & 13,276 & 61,610 \\
Average FTEs & 1.44 & 2.56 \\
$\quad$ Registered nurse & 1.00 & 1.50 \\
Registered practical nurse & $\begin{array}{c}\text { Insufficient } \\
\text { data }\end{array}$ & 2.80 \\
Advanced practice nurse & 0.97 & 1.48 \\
$\quad$ Nurse practitioner & 1.00 & 0.62 \\
Allied health care professionals & 0.90 & 2.25 \\
Administrative & & \\
$\quad$ support (eg, secretarial, clerk) & $72.70 \%$ & $63.63 \%$ \\
$\quad \begin{array}{l}\text { Percentage of programs } \\
\text { achieving wait-time }\end{array}$ & & \\
bench marks & & \\
\hline
\end{tabular}

improvement, both of which appear to be lacking within our current HF landscape.

We would like to highlight the following key survey findings, which are early opportunities for action, and should be supported by the CCS Guidelines knowledge translation apparatus and the CHFS:

1. There is heterogeneity in referral and discharge criteria across clinics, which may act as a barrier to accessing specialized HF care.

ACTION: Develop and disseminate more explicit guidance, both patient- and risk-based, on who should or should not be seen in an HFC, how often and by which modality.

2. There is significant reliance on nurse-dependent care models, without a clear health human resource strategy to inform how the growing burden of HF will be managed.

ACTION: Strike formal collaborations with other health care professional societies engaged in the care of patients with HF, to identify solutions that can inform a comprehensive long-term approach to health human resource policy development.

3. There is variability in ability to measure patient and system-related quality indicators and outcomes.

ACTION: Work with the CCS Quality Indicators Initiative to provide regular report cards on outcomes that reflect all components of the Institute of Health Improvement Quadruple Aim.

4. Critically, access to natriuretic peptide testing in Canada is not uniform.

ACTION: Identify and educate key policy leads in each health care region regarding adherence to best medical practice.

5. There is a lack of regional/provincial care maps and pathways to support the patient journey including transitions in care.

ACTION: Emulate and disseminate best practices that are already available in some Canadian regions and work collectively to iterate these for local context to optimize adoption. 
2. Howlett JG, Chan M, Ezekowitz JA, et al. The Canadian Cardiovascular Society Heart Failure Companion: bridging guidelines to your practice. Can J Cardiol 2016;32:296-310. across the care continuum.

ACTION: Partner with other national and regional agencies who have undertaken this work, to assess the impact on patient and system outcomes in HF.

7. There is need for a culturally sensitive and person-centric approach to care, including educational materials.

ACTION: Design, collaborate, house, and disseminate best-in-class multimodality educational materials.

The HFC staffing models and associated FTEs described in this survey provide background and a framework to inform a health human resource strategy to develop and retain a pipeline of highly qualified personnel, for a sustainable national HF action plan, and to guide local HF service delivery. Although it may be argued that drawing direct contrast with other countries is flawed due to inherent differences among health care systems, it remains true that compared with our international colleagues, HFCs in Canada are largely under-resourced by nurses, nurse practitioners, pharmacists, and physicians.

Variability in both access to HFCs and composition of the multidisciplinary team highlights the need for a standardized national approach to HF care in Canada. Several patient and organizational barriers to care were identified in this survey, which must inform the development of regional care models to improve transitions for this patient population. Unfortunately, we were unsuccessful in incorporating the perspectives of PCPs and internal medicine specialists who provide the majority of HF care in Canada, which in turn limits our ability to comment on strategies for capacity building outside the HFC setting. These considerations must be taken into account when interpreting our findings. That said, engaging all HF care providers, to build on the themes identified in this survey, will be an important next step in developing integrated and shared care models known to improve patient outcomes. $^{27}$

\section{Acknowledgements}

The authors thank the CHFS and CCS HF Panel members as well as our lived experience contributor Dr Sharon Bray for their participation and expertise. They also thank all of the HFCs in Canada who participated in the survey. The authors acknowledge the contributions of Dr Rohan Sanjanwala and Christianna Brooks (CCS Staff) for their assistance and expertise with survey development and analysis.

\section{Funding Sources}

There are no funding sources to disclose

\section{Disclosures}

The authors have no conflicts of interest to disclose.

\section{References}

1. Ezekowitz JA, O’Meara E, McDonald MA, et al. Comprehensive update of the Canadian Cardiovascular Society Guidelines for the management of heart failure. Can J Cardiol 2017;33:1342-433.
3. Jessup M, Albert NM, Lanfear DE, et al. ACCF/AHA/HFSA 2011 survey results: current staffing profile of heart failure programs, including programs that perform heart transplant and mechanical circulatory support device implantation: a report of the ACCF Heart Failure and Transplant Committee, AHA Heart Failure and Transplantation Committee, and Heart Failure Society of America. J Am Coll Cardiol 2011;57:2115-24.

4. McDonagh TA, Blue L, Clark AL, et al. European Society of Cardiology Heart Failure Association Standards for delivering heart failure care. Eur J Heart Fail 2011;13:235-41.

5. Minimal Requirements and Key Clinical Services for Heart Failure Programs Within a Spoke-Hub-Node Model of Care. Toronto, Ontario, Canada: CorHealth Ontario, 2018.

6. Quality Standards. Heart Failure: Care in the Community for Adults. Toronto, Ontario, Canada: Health Quality Ontario, March 2019.

7. Initiation of CPAP for newly diagnosed OSA in hospitalized heart failure patients. Available at: https://clinicaltrials.gov/show/NCT03056443. 2017. Accessed March 4, 2020.

8. Bui AL, Horwich TB, Fonarow GC. Epidemiology and risk profile of heart failure. Nat Rev Cardiol 2011;8:30-41.

9. Maggioni AP, Dahlstrom U, Filippatos G, et al. EURObservational research programme: regional differences and 1-year follow-up results of the Heart Failure Pilot Survey (ESC-HF Pilot). Eur J Heart Fail 2013;15: 808-17.

10. Ponikowski P, Anker SD, AlHabib KF, et al. Heart failure: preventing disease and death worldwide. ESC Heart Fail 2014;1:4-25.

11. The Burden of Heart Failure. The Heart and Stroke foundation, 2016. Available at: https://www.heartandstroke.ca/-/media/pdf-files/canada/2017heart-month/heartandstroke-reportonhealth-2016.ashx?la=en\&hash= 91708486C1BC014E24AB4E719B47AEEB8C5EB93E. Accessed May 3,2020

12. Kociol RD, Liang L, Hernandez AF, et al. Are we targeting the right metric for heart failure? Comparison of hospital 30-day readmission rates and total episode of care inpatient days. Am Heart J 2013;165:987-994. e1.

13. Cleland JGF, ftSGoDotWGoHFotESo Cardiology, Swedberg K, et al. The EuroHeart Failure survey programme- a survey on the quality of care among patients with heart failure in Europe: Part 1: patient characteristics and diagnosis. Eur Heart J 2003;24:442-63.

14. Ballard D, Ogola G, Fleming N, et al. Impact of a standardized heart failure order set on mortality, readmission, and quality and costs of care. Int J Qual Health Care 2010;22:437-44.

15. Van Spall HGC, Rahman T, Mytton O, et al. Comparative effectiveness of transitional care services in patients discharged from the hospital with heart failure: a systematic review and network meta-analysis. Eur J Heart Fail 2017;19:1427-43.

16. Van Spall HGC, Lee SF, Xie F, et al. Effect of patient-centered transitional care services on clinical outcomes in patients hospitalized for heart failure: the PACT-HF randomized clinical trial. JAMA 2019;321: 753-61.

17. Howlett JG. Specialist heart failure clinics must evolve to stay relevant. Can J Cardiol 2014;30:276-80.

18. Olano-Lizarraga M, Oroviogoicoechea C, Errasti-Ibarrondo B, SaracíbarRazquin M. The personal experience of living with chronic heart failure: 
a qualitative meta-synthesis of the literature. J Clin Nurs 2016;25: 2413-29.

19. Huitema AA, Harkness K, Heckman GA, McKelvie RS. The spoke-huband-node model of integrated heart failure care. Can J Cardiol 2018;34: 863-70.

20. Martinez AS, Saef J, Paszczuk A, Bhatt-Chugani H. Implementation of a pharmacist-managed heart failure medication titration clinic. Am J Health Syst Pharm 2013;70:1070-6.

21. Lowrie R, Mair FS, Greenlaw N, et al. Pharmacist intervention in primary care to improve outcomes in patients with left ventricular systolic dysfunction. Eur Heart J 2012;33:314-24.

22. Yancy CW, Jessup M, Bozkurt B, et al. 2013 ACCF/AHA guideline for the management of heart failure: a report of the American College of Cardiology Foundation/American Heart Association Task Force on practice guidelines. Circulation 2013;128:e240-327.

23. Virani SA, Bains M, Code J, et al. The need for heart failure advocacy in Canada. Can J Cardiol 2017;33:1450-4.
24. McMurray JJV, DeMets DL, Inzucchi SE, et al. The Dapagliflozin And Prevention of Adverse-outcomes in Heart Failure (DAPA-HF) trial: baseline characteristics. Eur J Heart Fail 2019;21:1402-11.

25. Ponikowski P, van Veldhuisen DJ, Comin-Colet J, et al. Beneficial effects of long-term intravenous iron therapy with ferric carboxymaltose in patients with symptomatic heart failure and iron deficiency. Eur Heart J 2015;36:657-68.

26. Maurer MS, Schwartz JH, Gundapaneni B, et al. Tafamidis treatment for patients with transthyretin amyloid cardiomyopathy. New Engl J Med 2018;379:1007-16.

27. Ezekowitz JA, van Walraven C, McAlister F, Armstrong P, Kaul P. Impact of specialist follow-up in outpatients with congestive heart failure. CMAJ 2005;172:189-94.

\section{Supplementary Material}

To access the supplementary material accompanying this article, visit CJC Open at https://www.cjcopen.ca/ and at https://doi.org/10.1016/j.cjco.2020.03.001. 